Research Group: Development

Septembre 2009

La qualité des institutions influence-telle la performance économique? Le cas des télécommunications dans les pays en voie de développement RABAH BELAID, FARID GASMI ET LAURA RECUERO VIRTO 


\title{
La qualité des institutions influence-t-elle la performance économique ? Le cas des télécommunications dans les pays en voie de développement*
}

\author{
Rabah BELAID \\ Ecole Nationale Supérieure de Statistique et d’Economie Appliquées (ENSSEA), Alger
}

Farid GASMI

Toulouse School of Economics (ARQADE \& IDEI), Université Toulouse 1 Capitole

\author{
Laura RECUERO VIRTO \\ Organisation de Coopération et de Développement Economiques (OCDE), Paris
}

\begin{abstract}
Résumé
De nombreuses études récentes ont montré que la qualité des institutions d’un pays est un facteur explicatif important de son développement économique. Des études empiriques ont mis en évidence une relation positive entre la qualité des institutions et la performance des politiques de réforme engagées dans certains secteurs clés des économies des pays en voie de développement. Le présent article se propose d'explorer cette relation pour le cas des télécommunications, un secteur parmi les industries d'infrastructure qui a connu de par le monde les changements structurels les plus profonds. Plus précisément, nous nous interrogeons sur la robustesse de cette relation aux différences de revenus qui existent au sein même des pays en voie de développement. Nous spécifions un modèle économétrique pour une base de données, couvrant la période 1985-1999, sur un échantillon de 32 pays en voie de développement que nous décomposons en deux panels selon le PIB par tête. Cette décomposition est effectuée dans le but de tester l'existence d'un seuil de revenu au-dessous duquel, une fois pris en compte l'effet des variables de réforme, notamment la libéralisation des marchés et la privatisation de l'opérateur historique, la qualité des institutions ne joue qu'un rôle marginal. Les résultats montrent qu'au contraire, l'impact de la qualité des institutions sur les performances de l'industrie est davantage perceptible dans les pays à faible PIB par tête. Ainsi, au sein même des pays en voie de développement, l'effet marginal d'un investissement consenti pour l'amélioration de la qualité des institutions est plus élevé dans les pays à plus faibles revenus. Ce résultat est en accord avec la tendance récente des bailleurs de fonds internationaux à soutenir des politiques d'aide économique de long terme privilégiant le renforcement des institutions dans les pays les moins développés.
\end{abstract}

\begin{abstract}
In recent years, a number of studies have shown that institutions are crucial to economic development. This literature has highlighted a positive relationship between the quality of the macro-institutional environment and the performance of reform policies conducted in some key sectors of the developing countries' economies. This paper explores this relationship for the case of telecommunications, the sector among the infrastructure industries that worldwide has probably experienced the most important structural changes. More specifically, we investigate the extent to which this relationship is robust to the level of development of these countries. We specify an econometric model for a data base of observations on a panel of 32 developing countries covering the period 1985-1999. The sample is decomposed into two panels according to GDP per capita, thus allowing us to test for the existence of a level of revenue below which, once the effect of reforms variables, in particular, market liberalization and privatization of the incumbent, has been controlled for, the quality of institutions plays only a minor role. We find that the impact of the institutional quality on the performance of the industry is more perceptible in the sample of countries with the lower GDP per capita. Thus, within the developing countries, the marginal effect of an investment in improving the institutional quality is higher in the countries with lower revenues. This result is in line with the recent trend of international donors to reallocate resources to long term policies for improving institutional mechanisms in less developed countries.
\end{abstract}

Septembre 2009

\footnotetext{
* Nous remercions A. Belkacem-Nacer, H. Hammoudi, P. Noumba et A. Zakane pour leurs suggestions sur une version préliminaire de ce travail. Cette version de l'article a largement bénéficié des commentaires de deux évaluateurs anonymes et de l'éditeur de cette revue. Toute erreur ou insuffisance qui subsisterait n’engage, néanmoins, que les auteurs.
} 


\section{Introduction}

Les politiques de développement préconisées par le consensus de Washington ont montré leurs limites dès le début des années 1990 et la réflexion s’est dès lors recentrée sur les facteurs institutionnels considérés de plus en plus comme étant la cible prioritaire des politiques d’aide au développement. Afin de démontrer le rôle vital que jouent les institutions dans le développement et la croissance économiques (Edison, 2003¹), de nombreuses études s'appuyant sur ce nouveau paradigme ont été menées mettant en évidence l'influence des environnements institutionnels sur l'efficacité des réformes sectorielles ${ }^{2}$. Une large part de ces études concerne les secteurs d'infrastructure, en particulier les télécommunications et l'énergie, traditionnellement structurés en monopoles publics intégrés verticalement.

Dans une approche conceptuelle et relevant davantage de la science politique, les déterminants clés d'une régulation sectorielle performante sont à trouver en amont de celleci, i.e., dans la "sphère politique" (Spiller et al, 2003, Spiller et Tommasi, 2004). Une approche plus empirique met l'accent sur la qualité de la gouvernance des instances de régulation en tant que déterminant clé des performances de la régulation sectorielle (Cubbin et Stern, 2005). Spiller et Liao (2008) présentent une revue de la littérature sur le rôle des groupes d'intérêt dans la définition des politiques économiques alors que Spiller et Tommasi (2007) s’intéressent aux fondements institutionnels des politiques publiques dans le cas de l'Argentine ${ }^{3}$.

Une littérature récente insiste sur l'intérêt d'orienter la discussion vers un cadre d’analyse intégré dans lequel aussi bien la qualité des instances de régulation sectorielle que celle de l'environnement global dans lequel fonctionnent ces instances sont prises en considération. Dans cette approche, la qualité des instances de régulation sectorielle apparaît comme une condition nécessaire mais non suffisante pour atteindre les objectifs assignés aux réformes sectorielles. L’argument à l'appui de cette assertion est qu'une bonne qualité de l'instance de régulation sectorielle peut être inhibée, voire stérilisée, par des facteurs de contre performance relevant de la sphère politique.

S’appuyant sur ce schéma d'analyse, Gasmi et al (2006) montrent par une étude économétrique que certaines caractéristiques décrivant la sphère politique, plus

\footnotetext{
${ }^{1}$ Le lecteur intéressé pourrait également consulter le chapitre III du document du FMI "Perspectives de l'économie mondiale", d'avril 2003.

${ }^{2}$ Un grand nombre d'analyses menées dans ce domaine portent sur les déterminants de la performance des systèmes de régulation sectorielle mis en place pour la conduite des réformes économiques.

${ }^{3}$ Ces analyses procèdent du paradigme de l’économie néo-institutionnelle.
} 
particulièrement la "responsabilisation politique" ${ }^{4}$, ont un impact significatif sur la performance de la régulation du secteur des télécommunications que ces auteurs mesurent par la performance économique de ce secteur ${ }^{5}$. L'existence d'un tel impact est montrée par une série de tests économétriques portant sur deux bases de données, une concernant un panel de pays développés et une autre un panel de pays en voie de développement. L’analyse montre alors que cet effet est plus fort pour les pays en voie de développement. Une implication de ce résultat est que l'effet marginal d'un effort d'amélioration de la qualité institutionnelle est significativement plus élevé dans les pays en voie de développement.

Cette étude fournit un double éclairage. D’une part, sur un plan méthodologique, elle montre l'intérêt d'une approche intégrée alliant les enseignements de l'analyse du rôle des institutions dans le développement économique et ceux de l'analyse de la performance de la régulation sectorielle. D’autre part, sur un plan plus pratique, les résultats auxquels elle aboutit éclairent le décideur sur les politiques économiques à mettre en œuvre pour améliorer la performance de la régulation sectorielle et, plus généralement, sur l'efficacité des politiques de réforme des industries d’infrastructure.

Une question que suggère cette étude est de savoir si, au sein même des pays en voie de développement, il n’existerait pas un "seuil critique de pauvreté" au-dessous duquel l'effet marginal d'un effort d'amélioration de la qualité des institutions deviendrait négligeable. L'existence d'un tel seuil suggèrerait que, pour les pays les moins avancés, l'amélioration de la qualité des institutions n'est pas un préalable nécessaire à leur prospérité. Elle impliquerait donc également que la relation mise en évidence dans la littérature récente, entre la qualité des institutions d’un pays et sa performance économique, n’est pas complètement indépendante du niveau de richesse du pays.

La présente étude se propose d'examiner cette question à l'aide d’une série de régressions ayant comme variables dépendantes des indicateurs de performance économique sectorielle et comme variables indépendantes des caractères mesurant la qualité des institutions. L'analyse porte sur deux sous échantillons de pays en voie de développement différenciés suivant le Produit Intérieur Brut par tête ${ }^{6}$. L'étude est menée à l'aide d'un modèle dynamique à effets fixes individuels spécifié suivant la méthode d’analyse des séries temporelles en coupes transversales. Les régressions des variables de performance du secteur

\footnotetext{
${ }^{4}$ Par "responsabilisation politique" nous entendons ici traduire le concept de political accountability qui rend compte de l'obligation pour l'acteur politique de répondre de ses actions. L'acteur est ainsi "comptable" de ses actes.

${ }^{5}$ Ces auteurs interprètent la performance du secteur des télécommunications comme la conséquence du processus de régulation.

${ }^{6}$ Le PIB par tête constitue un bon indicateur du niveau d'activité économique d’un pays.
} 
sur les indicateurs de qualité des institutions sont effectuées en tenant compte des variables de réformes qui, dans le contexte des pays en voie de développement, méritent une attention particulière. Les résultats obtenus militent fortement pour la mise en œuvre de politiques de renforcement des institutions dans les pays en voie de développement et ce, indépendamment du niveau de richesse de ces pays.

Cet article est organisé en cinq sections. La section qui suit décrit les variables du modèle économétrique et présente l’échantillon sur lequel porte l’analyse empirique. Une description succincte de la méthode économétrique utilisée est présentée dans la section 3. Les résultats des régressions représentant une estimation de la relation entre la qualité des institutions et la performance économique du secteur des télécommunications sont présentés et discutés dans la section 4. La dernière section résume les principaux résultats de l'analyse empirique, discute certaines de leurs implications en termes de politique économique et suggère quelques pistes de recherche pour approfondir la question. Des informations plus détaillées sur les variables du modèle (définition des échelles de mesure et sources des données pour chaque variable) ainsi que des tableaux statistiques présentant les résultats des traitements économétriques figurent en annexe.

\section{Variables et échantillons}

Notre analyse s'appuie sur une série de régressions ayant comme variable dépendante une mesure de la performance économique du secteur des télécommunications et comprenant dans l'ensemble des variables indépendantes une mesure de la qualité des institutions. Ces régressions sont effectuées en tenant compte de l'effet des variables de réforme, en l’occurrence, la libéralisation des marchés et la privatisation de l’opérateur historique.

D’autres variables de contrôle sont introduites, en particulier, pour tenir compte de certains effets spécifiques aux pays et de l’influence du mode de structuration de la régulation sectorielle.

La présente section décrit, dans les sous-sections 2.1, 2.2 et 2.3, l'ensemble de ces variables. Celles-ci sont récapitulées dans le Tableau 1 ci-dessous. Cette section présente également, dans la sous-section 2.4, la structure de l'échantillon sur lequel ces variables sont mesurées. 


\subsection{Variables dépendantes}

La "performance" du secteur des télécommunications est saisie à travers le niveau d'offre de service (output), l'efficience productive et le niveau des prix. Le premier caractère est mesuré par la télédensité du réseau de téléphonie fixe (le nombre de lignes fixes pour 100 habitants) et le nombre d’abonnés aux services de téléphonie mobile. L'efficience productive est mesurée par le nombre de lignes par employé dans le segment de la téléphonie fixe. Quant au niveau des prix, nous différencions la charge mensuelle d’accès au réseau filaire du prix d'usage du téléphone mobile. La performance du secteur d’intérêt est donc mesurée par cinq variables : la télédensité du fixe (réseau filaire), le nombre d’abonnés aux services de téléphonie mobile (réseau cellulaire), le prix du service de téléphonie fixe, le prix du service de téléphonie mobile et le nombre de lignes téléphoniques par employé.

\subsection{Variables indépendantes}

Un examen de la littérature sur la notion d’"environnement institutionnel"7 permet de définir ce dernier comme "l’organisation des relations qui existent entre un certain nombre de dispositifs structurant ses capacités internes de transformation". L'environnement institutionnel d'un pays est ainsi perçu comme un ensemble composé de cinq éléments, à savoir, les institutions législatives et exécutives, les institutions judiciaires, les capacités administratives, la nature des conflits d'intérêt et les coutumes et normes informelles. Bien que jouant un rôle important, ces deux dernières composantes de l'environnement institutionnel sont les plus difficilement formalisables et les analyses empiriques récentes de l’influence des institutions sur les performances économiques mettent davantage l'accent sur les trois premiers éléments de cet ensemble. Celles-ci retiennent généralement trois caractères pour apprécier la qualité institutionnelle : i) les pratiques de gestion des affaires publiques (corruption, droits politiques, poids de la réglementation, etc.) ; ii) l'existence ou non de lois protégeant la propriété privée et le degré d’observance de la Loi en général par les acteurs; iii) les contraintes imposées aux dirigeants politiques par les dispositifs de contrôle et d'équilibre des pouvoirs.

Dans la présente étude, la "qualité des institutions" est principalement définie par la qualité du processus politique et la qualité de l'environnement institutionnel. Le premier caractère est mesuré par une variable ordinale qui renseigne sur la répartition et l'équilibre

\footnotetext{
${ }^{7}$ Voir par exemple Levy et Spiller (1994) et North (1991).
} 
des pouvoirs ${ }^{8}$ législatif, exécutif et judiciaire. Le deuxième caractère est mesuré à partir de cinq variables indicatrices du degré de corruption, de la force du système judiciaire et le degré d’observance des lois, de la qualité de l'appareil administratif de l'Etat, du risque d'expropriation des investisseurs privés et du risque de change ${ }^{9}$.

Les variables reflétant la qualité des institutions sont, à l'évidence, celles dont la mesure est la moins aisée. Dans le cas qui nous intéresse, aux difficultés habituelles que rencontre l'économètre lorsqu'il s'agit de quantifier des caractères qualitatifs s'ajoute le risque d'erreur de mesure associé à une définition restrictive des institutions. Par ailleurs, la consistance de ces données qui concernent différents pays n'est à l'évidence pas garantie, ce qui rend délicate la comparabilité interrégionale du "contenu" des variables. En dépit des précautions prises et des efforts considérables consentis pour la construction de la base de données, il importe donc de garder à l'esprit ces contraintes lors de l'interprétation des résultats.

\subsection{Variables de contrôle}

L’introduction de variables de contrôle dans les régressions permet d'éviter un éventuel biais dû à l'omission de variables ayant un effet sur la performance économique du secteur des télécommunications. A cet effet, trois variables reflétant l'avancée de la "réforme" du secteur sont introduites dans le modèle de régression. La première rend compte du degré de privatisation (taux d'ouverture du capital) de l'opérateur historique. La deuxième est une indicatrice de la structure du marché de la téléphonie fixe (monopole vs marché concurrentiel). La troisième variable reflète le degré de concurrence sur le marché de la téléphonie mobile.

Nous avons également introduit comme "autres" variables de contrôle la densité de la population globale, le poids relatif de la population rurale et le mode de structuration de la régulation sectorielle. Les deux premières variables permettent de tenir compte d'un facteur qui revêt un caractère particulièrement important dans les industries d'infrastructure, à savoir, la distribution spatiale des populations. En effet, le coût d'implémentation et la rentabilité des infrastructures sont fortement dépendants de cette distribution. La troisième est une variable indiquant s’il existe une instance dédiée à la régulation sectorielle.

\footnotetext{
${ }^{8}$ Au sens de De l'esprit des lois de Montesquieu.

${ }^{9}$ Il convient de noter que cette variable de risque de change ne reflète qu'indirectement la qualité des institutions financières d'un pays.
} 
Le Tableau 1 ci-dessous récapitule l'ensemble des variables sur lesquelles porte l'analyse empirique et qui sont regroupées en 4 catégories, à savoir: "Performance", "Qualité des institutions", "Reforme" et "Autres".

Tableau 1

Variables du modèle*

\begin{tabular}{|c|c|c|}
\hline & Symboles & Intitulés des variables \\
\hline \multirow{5}{*}{ 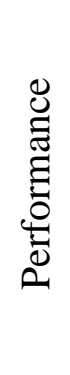 } & tele-fix & Télédensité du réseau fixe \\
\hline & mobil & Nombre d'abonnés aux services de téléphonie mobile \\
\hline & p-mobil & Prix du service de téléphonie mobile \\
\hline & $p$-fix & Prix du service de téléphonie fixe \\
\hline & l-empl & Nombre de lignes téléphoniques par employé \\
\hline \multirow{7}{*}{ 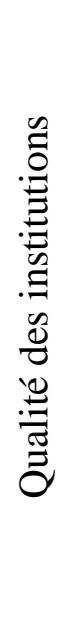 } & pouvoir & $\begin{array}{l}\text { Qualité du processus politique (Equilibre des } \\
\text { pouvoirs) }\end{array}$ \\
\hline & Inst & Qualité de l'environnement institutionnel \\
\hline & corrup & Degré de corruption \\
\hline & Loi & $\begin{array}{l}\text { Force du système judiciaire et degré d'observance des } \\
\text { lois }\end{array}$ \\
\hline & $A d m$ & Qualité de l'appareil administratif de l'Etat \\
\hline & exprop & Risque d'expropriation \\
\hline & Change & Risque de change \\
\hline \multirow{3}{*}{ 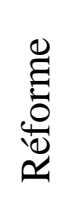 } & Privat & Degré de privatisation de l’opérateur historique \\
\hline & concur-fix & Concurrence (ou non) sur le marché du fixe \\
\hline & concur-mobil & Degré de concurrence sur le marché du mobile \\
\hline \multirow{3}{*}{ 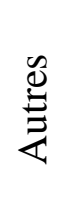 } & dens-pop & Densité de la population globale \\
\hline & pop-rural & Poids de la population rurale \\
\hline & sep-regul & Séparation (ou non) du régulateur \\
\hline
\end{tabular}

*Les échelles de mesure de chacune de ces variables sont décrites dans la section B de l’annexe. 


\subsection{Structure de l’échantillon}

Les variables du modèle sont mesurées sur 32 pays en voie de développement classés dans deux sous-échantillons selon le PIB par tête exprimé en Dollars US de 2000, de part et d'autre de la valeur médiane de ce ratio qui est de 1.561 Dollars US en moyenne, sur la période couverte par l'analyse ${ }^{10}$.

La partition de l'échantillon en deux panels est faite de manière exogène pour pouvoir effectuer des comparaisons entre ces deux groupes de pays et tester l'hypothèse formulée dans la section précédente. En procédant ainsi, notre but est de voir si, en utilisant comme critère de séparation le "niveau d'activité économique d'ensemble ${ }^{11}$ ", les variables "institutionnelles" agissent différemment sur la performance économique du secteur d'intérêt, en passant d'un sous échantillon à l'autre ${ }^{12}$.

Le premier groupe, composé de 16 pays dont le PIB par tête est supérieur à cette valeur médiane, est désigné dans la suite par "Panel 1" ou "Pays à revenus intermédiaires". Les 16 autres pays, pour lesquels le PIB par tête est au-dessous de la valeur médiane, forment le "Panel 2" ou "Pays à faibles revenus". Ces deux échantillons couvrent la période allant de 1985 à $1999^{13}$.

La liste des pays ainsi répartis apparaît dans la Figure 1 ci-dessous. Cette figure représente la distribution des 32 pays suivant le même critère de classification (PIB/tête) mais avec des données couvrant la période 2000-2005 qui est postérieure à la période sur laquelle porte l'analyse empirique, à savoir 1985-1999. Entre ces deux périodes, le PIB par tête médian est passé de 1.561 à 1.951 Dollars US (aux prix constants de 2000) mais, à une exception près ${ }^{14}$, la partition de l'échantillon est restée inchangée dans cette translation temporelle. Ces résultats montrent que les positions relatives des pays par rapport à la valeur médiane du PIB par tête persistent durant les deux décennies que couvre la période 19852005, ce qui conforte le choix de ce critère dans la construction des deux panels.

\footnotetext{
${ }^{10}$ Cette période va de 1985 à 1999.

${ }^{11}$ Le PIB par tête constituant en effet un bon indicateur du niveau d'activité économique d'ensemble.

${ }^{12}$ Une approche alternative aurait consisté, ce qui aurait considérablement alourdi la procédure économétrique, à utiliser l'échantillon global et estimer les "effets croisés" des variables institutionnelles avec une variable indicatrice (dummy) du niveau du PIB par tête.

${ }^{13}$ Compte tenu du problème des données manquantes et afin d'exploiter au mieux l'ensemble des données disponibles, les deux panels étudiés sont non cylindrés.

${ }^{14}$ Il s'agit d'une permutation entre la Thaïlande et la Jordanie.
} 


\section{Figure 1}

Positionnement des pays suivant le niveau moyen et la croissance annuelle du

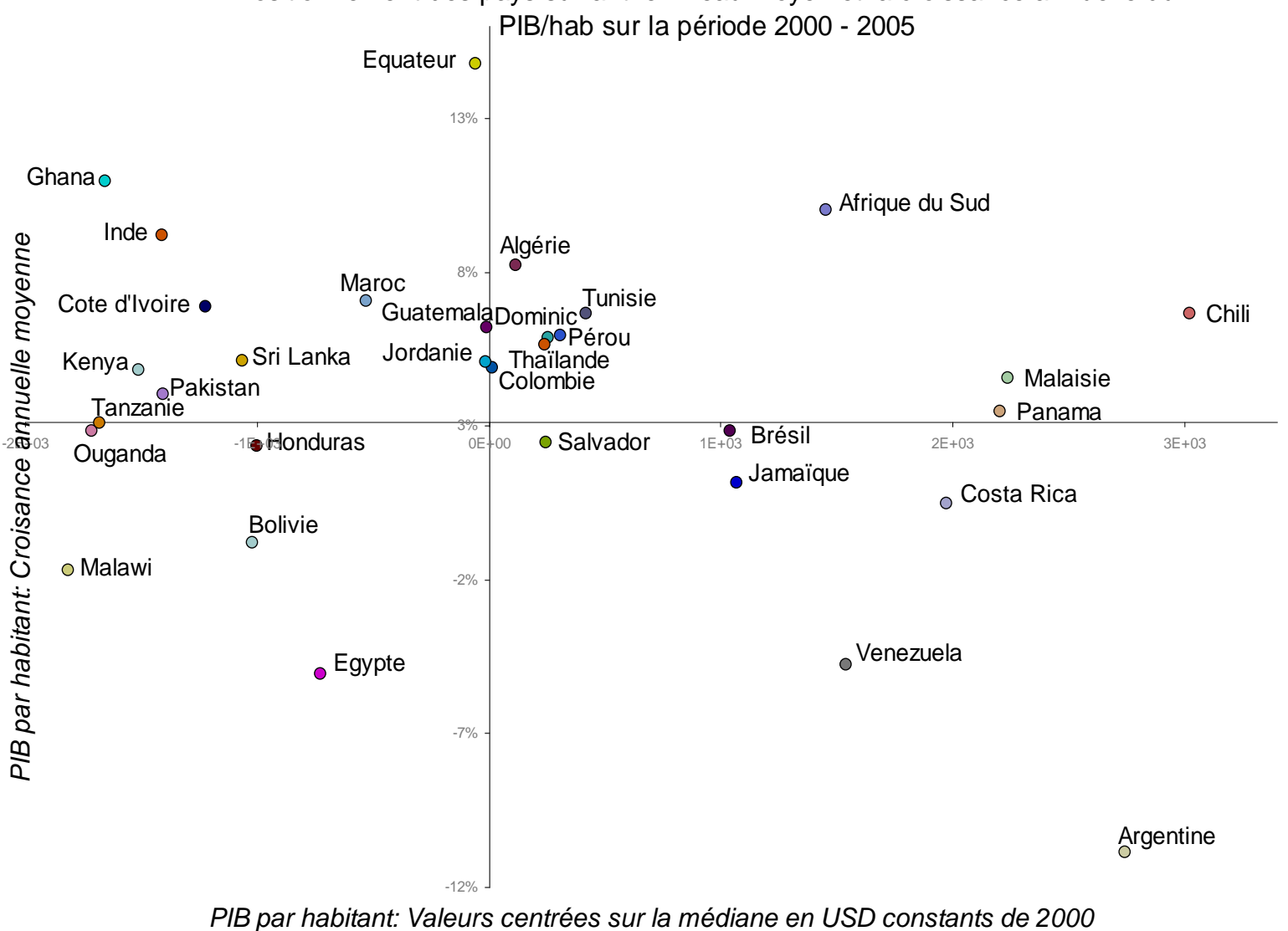

\section{Méthode économétrique}

Cette section présente les ingrédients essentiels de la méthode économétrique utilisée pour estimer la relation entre la qualité institutionnelle et la performance économique du secteur des télécommunications dans les deux panels décrits précédemment. Comme ces données combinent les aspects d'une série temporelle et ceux d'une coupe instantanée ${ }^{15}$, elles sont analysées à l'aide de la Méthode des Moments Généralisés en Différences ${ }^{16}$ (DIF-GMM) développée par Arellano et Bond (1991) pour les données de panel et adaptée récemment par Beck et Katz (2004) pour les données TSCS. L’équation suivante est spécifiée :

$$
\ln \left(y_{i t}\right)=\alpha_{0}+\alpha_{1} \ln \left(y_{i t-1}\right)+\mathrm{x}_{i t}^{\prime} \beta+\mu_{i}+\varepsilon_{i t}
$$

\footnotetext{
${ }^{15}$ Ce type de données est désigné par les termes de Time-Series-Cross-Sectional data (TSCS).

${ }^{16}$ L'acronyme DIF-GMM correspond à Differenced Generalized Method of Moments.
} 
où $y$ est une variable dépendante unidimensionnelle ${ }^{17}$ représentant la performance de la régulation dans le secteur des télécommunications, $\alpha_{0}$ et $\alpha_{1}$ sont des paramètres unidimensionnels inconnus, $\mathrm{x}$ est un vecteur de variables indépendantes représentant, entre autres, la qualité des institutions, $\beta$ est le vecteur des paramètres associés à ces variables, $\mu$ est un paramètre représentant l'effet fixe du modèle et $\varepsilon$ est un terme d'erreur. Les indices $i=1,2, \ldots, N$ et $t=1,2, \ldots, T$ indiquent le pays et l'année, respectivement. Les termes d'effet fixe et d'erreur satisfont les hypothèses habituelles $\mathrm{E}\left(\mu_{i}\right)=\mathrm{E}\left(\varepsilon_{i t}\right)=\mathrm{E}\left(\varepsilon_{i t} \mu_{i}\right)=\mathrm{E}\left(y_{i t} \varepsilon_{i t}\right)=0$.

L'estimation directe de l'équation (1) expose l'économètre à un problème de biais d'endogèneité dû à une corrélation de deux types : i) une corrélation entre une des variables indépendantes et le terme représentant l'effet fixe ; ii) une corrélation entre une des variables indépendantes et le terme d'erreur. Une façon d'éliminer les conséquences d'une corrélation du premier type est de transformer les variables de l'équation (1) en leurs différences premières et d'estimer

$$
\Delta \ln \left(y_{i t}\right)=\alpha_{1} \Delta \ln \left(y_{i t-1}\right)+\Delta \mathrm{x}_{i t}^{\prime} \beta+\Delta \varepsilon_{i t}
$$

où $\Delta$ est l'opérateur de différence première. Cependant, cette transformation pourrait générer un autre problème d'endogèneité provenant d'une corrélation contemporaine entre $\ln \left(y_{i t-1}\right)$ et le terme $\varepsilon_{i t-1}$. Comme cette corrélation est de même nature que celle du deuxième type mentionnée ci-dessus, la question est alors de trouver des variables instrumentales utilisables pour l'estimation de l'équation (2). La procédure appropriée, développée par Anderson et Hsiao (1981 et 1982) et généralisée par Arellano et Bond (1991), consiste à utiliser comme instruments les différences premières retardées de la variable endogène.

Avant d'estimer la relation entre la qualité des institutions et la performance du secteur, deux aspects des variables sont examinés : leur stationnarité et l'existence de liens de causalité entre elles. La motivation pour l'investigation de la stationnarité des variables est que, dans le contexte de DIFF-GMM et sous certaines conditions, la procédure d'instrumentation des variables endogènes est sujette au problème de faiblesse des

\footnotetext{
${ }^{17}$ L'équation (1) est estimée de manière contingente pour chacun des cinq indicateurs de performance définis dans la section 2 .
} 
instruments ${ }^{18}$. Cette difficulté peut être contournée en transformant, lorsque c'est nécessaire, les variables en leurs différences successives pour les rendre stationnaires ${ }^{19}$. Quant à la recherche de relations de causalité, la mise en évidence empirique de telles relations donne considérablement plus de consistance à l'interprétation de l'impact quantitatif du contexte institutionnel général sur la performance économique du secteur des télécommunications.

La question de la stationnarité des variables est analysée à l'aide d'une méthode proposée par Blundell et Bond $(1998,1999)$ dont les éléments se retrouvent également chez Arellano et Bover (1995). Celle de la causalité est traitée à l'aide d'une méthode qui combine DIFF-GMM et une procédure de tests de causalité au sens de Granger, développée par Holtz Eakin et al. (1988) pour des données de Panel. Ces tests sont basés sur l'estimation d'une équation de la forme

$$
\Delta \ln y_{i t}=\sum_{k=1}^{m} \alpha_{k} \Delta \ln y_{i t-k}+\sum_{k=1}^{m} \delta_{k} \Delta x_{i t-k}+\Delta \mathrm{x}_{i t}^{\prime} \beta+\Delta \varepsilon_{i t}
$$

Cette équation est utilisée pour tester si une variable représentant la qualité des institutions, $x$, "cause au sens de Granger" la variable représentant la performance de la régulation, $y$.

L’analyse de la stationnarité des variables ${ }^{20}$ permet également de se prononcer sur la validité des variables instrumentales. Les Tableaux I et II figurant dans la section C de l'annexe présentent les résultats de l'estimation d'un modèle autorégressif d'ordre 1 , AR(1), par DIF-GMM et par SYS-GMM ${ }^{21}$, une méthode qui utilise, en plus des conditions de moments prises en compte dans DIF-GMM, des instruments en différences premières pour l'équation en niveaux. Ces deux méthodes sont appliquées aux cinq indicateurs de la performance économique sectorielle en niveaux pour vérifier leur stationnarité. Dans le cas où la stationnarité n’est pas vérifiée par l'application de DIFF-GMM aux variables en niveaux, la dernière colonne de chacun des Tableaux I et II présente les résultats de la méthode DIF-GMM appliquée à ces variables en différences premières.

\footnotetext{
${ }^{18}$ Alonso-Borrego et Arellano (1996) ainsi que Blundell et Bond (1998) montrent que si les variables en différences premières sont faiblement corrélées avec leurs valeurs retardées en niveaux, les instruments disponibles pour les équations en différences premières sont faibles.

${ }^{19}$ La transformation des variables pour les rendre stationnaires améliore sensiblement la précision des estimateurs.

${ }^{20}$ Il s'agit ici de la stationnarité des séries temporelles autour d'un terme de tendance (time trend). Excepté pour la variable de productivité (lignes par employé), l'introduction de ce terme améliore en effet la significativité d'ensemble (goodness-of- fit) du modèle.

${ }^{21}$ L'acronyme SYS-GMM correspond à System Differenced Generalized Method of Moments.
} 
Ces tableaux contiennent les estimations du coefficient du processus AR(1) par DIFGMM et SYS-GMM, du coefficient associé au terme de tendance (Trend), des coefficients d'auto-corrélation de premier ordre $\left(m_{1}\right)$ et de second ordre $\left(m_{2}\right)$, des résidus en différences premières, la statistique permettant de tester la validité des instruments ( $J$-stat), la valeur initiale de décalage des instruments $(L)$ et le nombre d’observations effectivement utilisées (Nombre Obs).

Les résultats de l'estimation du modèle AR (1) montrent que les séries en niveaux des tarifs du cellulaire (p-mobil) et du fixe ( $p$-fix) et les séries en différences premières des trois autres indicateurs de performance économique, à savoir, la télédensité du fixe (tele-fix), le nombre d'abonnés au mobile (mobil) et le nombre de lignes par employé dans le fixe ( $l$ emp) sont stationnaires. Les résultats montrent également que l'hypothèse d'auto-corrélation de second ordre des résidus en différences premières est rejetée dans toutes les estimations du processus $\operatorname{AR}(1)^{22}$. De plus, les valeurs de $J$-stat ne rejettent pas l'hypothèse de validité des instruments. Ces résultats suggèrent que les variables en niveaux décalés de deux périodes et plus ainsi que les variables en différences premières retardées d'une période constituent des instruments valides.

Nous examinons à présent les résultats sur l'existence de relations causales entre les variables reflétant la qualité des institutions et les indicateurs mesurant la performance économique du secteur des télécommunications ${ }^{23}$. Les résultats de l'estimation par la méthode DIF-GMM intégrée à des tests de causalité telle qu'elle a été décrite ci-dessus sont présentés dans la section $\mathrm{C}$ de l'annexe. Ceux-ci révèlent une absence de liens de causalité pour les pays du Panel 1. En revanche, des liens de causalité existent dans le Panel 2 (voir le Tableau III), en particulier, lorsque la performance économique du secteur des télécommunications est mesurée par le nombre d'abonnements ou par le tarif des communications sur le segment du cellulaire. Quels enseignements peut-on tirer de ces résultats sur l'existence de relations causales dans les deux panels qui est indiquée dans le Tableau $2^{24}$ ?

\footnotetext{
${ }^{22}$ En effet, pour les deux panels et les cinq variables dépendantes, la valeur du coefficient $m_{2}$ est statistiquement non significative au seuil de $5 \%$.

${ }^{23}$ La relation entre la qualité des institutions et la performance du secteur fera l'objet d'un examen plus détaillé dans la prochaine section.

${ }^{24}$ Dans ce tableau, un "Oui" indique qu'une relation causale a été trouvée pour la paire de variables représentant la performance sectorielle et la qualité des institutions, alors qu'un "Non" signifie qu'une telle relation n’a pas pu être mise en évidence dans les données par la procédure de test de Granger.
} 
Tableau 2

Relations de causalité

(Panel 1, Panel 2)

\begin{tabular}{ccc}
\hline \hline \multirow{2}{*}{ Performance sectorielle } & \multicolumn{2}{c}{ Qualité des institutions } \\
& Equilibre des pouvoirs & Environnement institutionnel \\
\hline tele-fix & (Non, Non) & (Non, Non) \\
mobil & (Non, Oui) & (Non, Oui) \\
$l$-emp & (Non, Non) & (Non, Non) \\
$p$-fix & (Non, Non) & (Non, Non) \\
$p$-mobil & (Non, Non) & (Non, Oui) \\
\hline
\end{tabular}

Il convient d'abord de souligner que l'existence même d'un contraste entre les résultats obtenus pour les deux panels justifie ex post la stratification de notre ensemble de pays en voie de développement en deux sous-ensembles suivant le critère du revenu médian par habitant. Il faut ensuite remarquer que ces résultats sont en accord avec l'idée que l'utilité marginale d'un investissement pour l'amélioration du cadre institutionnel est relativement plus élevée dans les pays à plus faibles revenus. Enfin, il ne faudrait pas négliger le fait que l'absence d'"évidence" statistique sur l'existence de relations causales pour le panel 1 pourrait être due à la "sévérité" de la procédure de test de causalité. Au vu de ces commentaires et de l'importance d'une évaluation empirique de la relation entre la qualité des institutions et la performance économique dans le secteur des télécommunications, notamment dans les pays les plus pauvres, il est opportun d'effectuer les régressions pour les deux panels.

Cependant, afin d'éviter un éventuel biais d’omission de variable, ces régressions sont effectuées en tenant compte des effets d'un certain nombre de facteurs sur la performance économique du secteur. Ces variables de contrôle ont été discutées dans la sous-section 2.3. Parmi ces variables, celles reflétant l'avancée des réformes ont été instrumentées lorsqu'il s’est avéré qu’elles étaient endogènes. Les régressions sont effectuées en tenant compte également de l'effet d'évènements spécifiques conjoncturels, saisi par des variables indicatrices du temps. Les résultats des régressions sont présentés et discutés dans la section qui suit.

\section{Qualité des institutions et performance du secteur des télécommunications}

Nous discutons à présent les résultats des estimations de la relation entre la qualité des institutions et la performance économique du secteur des télécommunications. Nous 
présentons successivement les résultats relatifs aux pays à revenus intermédiaires du Panel 1 (sous-section 4.1) et ceux relatifs aux pays à faibles revenus du Panel 2 (sous-section 4.2).

\subsection{Pays à revenus intermédiaires}

Dans le groupe de pays à revenus intermédiaires, le Panel 1, les régressions mettent en évidence un effet positif de la qualité de l'environnement institutionnel sur la performance économique du secteur des télécommunications lorsque cette variable est mesurée par le tarif du fixe. Le Tableau 3 montre en effet que l'amélioration de la qualité de l'environnement institutionnel entraîne une baisse du tarif du fixe.

Lorsque la qualité des institutions est mesurée par les quatre autres indicateurs de performance, les résultats n'indiquent pas de liens significatifs. Cependant, les variables indicatrices du temps produisent un effet significatif sur le nombre de lignes par employé, le nombre d'abonnés au cellulaire ainsi que sur le tarif du fixe. La significativité des indicatrices du temps révèle que la performance économique du secteur est largement affectée par des événements spécifiques conjoncturels. La faiblesse des résultats concernant l'impact des variables institutionnelles dans le Panel 1 s'expliquerait donc par la prédominance de ces événements conjoncturels, ce qui semble cohérent avec l'absence de causalité entre les variables institutionnelles et la performance économique du secteur d'intérêt (voir le Tableau 2).

Qu'en est-il des variables de réformes ? Les résultats présentés dans le tableau 3 montrent que le degré d'ouverture du capital de l'opérateur historique influe positivement sur la performance économique du secteur. Cette relation est mise en évidence lorsque la performance sectorielle est mesurée par la télédensité du fixe, le nombre d'abonnés aux services de téléphonie mobile et le nombre de lignes par employé. La privatisation apparait ainsi comme un facteur d'amélioration des performances économiques du secteur des télécommunications dans le groupe des pays à revenus intermédiaires.

Quant à l’augmentation du degré de concurrence sur le marché du fixe, elle se traduit par une baisse de la télédensité et du nombre de lignes par employé dans ce même segment de marché. Ce résultat peut être mieux interprété en tenant compte du segment de la téléphonie mobile où l'on observe un impact positif de la concurrence sur le nombre d'abonnés. La baisse de la télédensité du fixe peut, en effet, s'expliquer par un effet de transfert de la demande vers le marché de la téléphonie mobile, devenu plus attractif du fait de l'intensification de la concurrence et de la diversification subséquente de l'offre sur ce segment de marché. 
Cet effet de transfert constitue également un argument permettant d'expliquer la baisse du nombre de lignes par employé. En effet, une diminution de la demande sur le marché du fixe conduit vraisemblablement à un abandon des lignes devenues non rentables du fait de la diminution de la télédensité sur ce marché. Or, cette baisse du nombre de lignes n’entraine pas forcément une réduction du nombre d'employés si l'on tient compte du fait

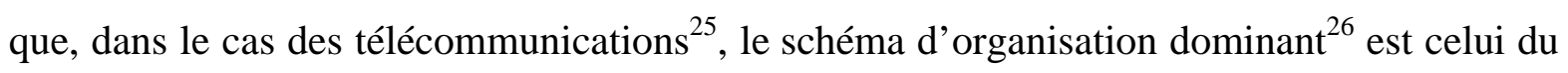
monopole public intégré verticalement. La rigidité de l'emploi (observable dans ce type d'organisation) combinée à une baisse du nombre des lignes exploitées permet ainsi d'expliquer l’impact négatif de la concurrence sur le nombre de lignes par employé.

Concernant les autres variables de contrôle, trois résultats méritent d’être soulignés à la lecture du Tableau 3. D’abord, l'accroissement de la densité démographique produit un effet positif sur le nombre de lignes par employé. Ce résultat reflète la réduction de la taille minimale d'efficacité du réseau ${ }^{27}$ filaire, induite par une plus forte concentration géographique des abonnés. Ensuite, cette même variable de densité démographique entraîne une augmentation du tarif du fixe. Ce résultat, a priori contre-intuitif, peut néanmoins s'expliquer si on tient compte des signes positifs ainsi que des valeurs élevées des paramètres associés aux variables indicatrices du temps ${ }^{28}$. Ces indications dénotent en effet une nette prédominance de l'effet d'événements conjoncturels dans l'explication de la hausse du tarif du fixe. Enfin, l’accroissement de la population rurale affecte "négativement" la télédensité du fixe et le nombre de lignes par employé, de même qu'il entraine une hausse du tarif du fixe. Ce résultat s’explique bien par le fait qu'une prédominance de la population rurale détermine, en toute vraisemblance, une demande éparse, impliquant un faible maillage territorial par le réseau filaire, d'où l'impact négatif sur les variables d'accès aux services (télédensité et tarif du fixe) et de productivité du travail (lignes par employé).

En résumé, les résultats montrent que le lien entre la qualité des institutions et la performance économique du secteur des télécommunications n’apparait que très faiblement dans le groupe des pays à revenus intermédiaires. La performance de ce secteur est mieux corrélée aux variables de réformes, aux facteurs démographiques ainsi qu’à l’occurrence d'événements conjoncturels.

\footnotetext{
${ }^{25}$ A l'instar des autres industries d'infrastructure.

${ }^{26}$ Notamment dans les pays en voie de développement.

${ }^{27}$ Impliquant un plus faible besoin en facteurs de production, dont le nombre d'employés.

${ }^{28}$ Voir le tableau IV-a de la section D de l'annexe.
} 
Tableau 3

Impact de la qualité des institutions sur la performance sectorielle (Panel 1 : pays à revenus intermédiaires) ${ }^{29}$

\begin{tabular}{|c|c|c|c|c|c|c|c|c|c|c|c|c|}
\hline & & & \multicolumn{10}{|c|}{ Variables dépendantes (Indicateurs de performance) } \\
\hline \multicolumn{3}{|c|}{ Variables indépendantes } & \multicolumn{2}{|c|}{ tele-fix } & \multicolumn{2}{|l|}{ mobil } & \multicolumn{2}{|l|}{ l-emp } & $p$-fix & \multicolumn{2}{|c|}{$p-m b l$} & \\
\hline \multicolumn{3}{|c|}{ Equilibre des pouvoirs } & \multicolumn{2}{|c|}{ NS } & \multicolumn{2}{|l|}{ NS } & \multicolumn{2}{|l|}{ NS } & NS & \multicolumn{2}{|c|}{ NS } & \\
\hline \multicolumn{3}{|c|}{ Environnement institutionnel } & \multicolumn{2}{|c|}{ NS } & \multicolumn{2}{|l|}{ NS } & \multicolumn{2}{|l|}{ NS } & - & \multicolumn{2}{|c|}{ NS } & \\
\hline & & privat & \multicolumn{2}{|l|}{+} & \multicolumn{2}{|l|}{+} & \multicolumn{2}{|l|}{+} & NS & \multicolumn{2}{|c|}{ NS } & \\
\hline & & cur-fix & \multicolumn{2}{|l|}{-} & \multicolumn{2}{|l|}{ NS } & \multicolumn{2}{|l|}{-} & NS & \multicolumn{2}{|c|}{ NS } & \\
\hline & conc & -mobil & \multicolumn{2}{|c|}{ NS } & \multicolumn{2}{|l|}{+} & \multicolumn{2}{|l|}{ NS } & NS & \multicolumn{2}{|c|}{ NS } & \\
\hline & & -regul & \multicolumn{2}{|c|}{ NS } & \multicolumn{2}{|l|}{ NS } & \multicolumn{2}{|l|}{ NS } & NS & \multicolumn{2}{|c|}{ NS } & \\
\hline & & ns-pop & \multicolumn{2}{|c|}{ NS } & \multicolumn{2}{|l|}{ NS } & + & & + & & & \\
\hline & & o-rural & - & & NS & & - & & + & & & \\
\hline & formes & dogènes & Oui & & Oui & & Oui & & Oui & & & \\
\hline & & & & Indica & trices du & tem! & & & & & & \\
\hline $\begin{array}{c}\text { Variables } \\
\text { Dépendantes }\end{array}$ & 1988 & 89 & 90 & 91 & 92 & 93 & 94 & 95 & 96 & 97 & 98 & 99 \\
\hline tele-fix & NS & NS & NS & - & NS & NS & NS & - & NS & NS & - & - \\
\hline Mobil & + & + & + & NS & + & + & NS & + & NS & NS & NS & + \\
\hline$p$-fix & NS & NS & NS & NS & + & + & + & + & + & + & + & + \\
\hline
\end{tabular}

\subsection{Pays à faibles revenus}

Un premier résultat significatif mis en évidence dans le groupe de pays à faibles revenus, le Panel 2, est qu'une meilleure qualité de l'environnement institutionnel améliore la performance économique du segment de la téléphonie mobile. Le tableau 4 montre, en effet, que l'amélioration de cette variable institutionnelle entraine un accroissement du nombre d'abonnements et une baisse du tarif sur le marché de la téléphonie mobile. Ce résultat confirme la robustesse du lien de causalité déjà établi à l’aide des tests de Granger (voir le Tableau 2).

Sur le segment du fixe, l'amélioration de la qualité de l'environnement institutionnel produit des effets contrastés. Cette amélioration conduit à une augmentation du nombre de lignes par employé, reflétant une amélioration de la productivité du travail sur le segment du fixe. Elle conduit également à une augmentation du tarif du fixe, reflétant une détérioration de l'accessibilité des abonnés aux services sur ce même segment de marché. Si le premier effet correspond bien au résultat attendu, le deuxième, qui peut paraître surprenant, peut être mis sur le compte des politiques de rééquilibrage des tarifs mises en œuvre durant la période

${ }^{29}$ Les résultats chiffrés concernant ce groupe de pays figurent au Tableau IV-a en Annexe (Section D). 
étudiée. On retient que, globalement, dans le groupe des pays les moins avancés, une bonne qualité de l'environnement institutionnel produit des effets plutôt bénéfiques sur la performance économique du secteur des télécommunications.

Par contre, la qualité du processus politique, représentée par la variable d’équilibre des pouvoirs, est peu significative. Le seul impact significatif porte sur le nombre d'abonnés au cellulaire avec un signe négatif, contraire au signe attendu dans cette relation. Ce résultat est sans doute lié, plus en amont, à la contrainte informationnelle telle que nous l'avons brièvement discutée dans la sous-section 2.3. Outre la difficulté de produire une mesure fiable de la qualité du processus politique, il convient aussi de s'interroger, dans le cas des pays les moins avancés, sur le fonctionnement effectif des instances représentant les pouvoirs législatif et judiciaire ainsi que sur la manière dont ces instances peuvent agir face au pouvoir exécutif. Dans les pays sous développés, la prédominance des transactions informelles au sein de la sphère politique est de nature à altérer fortement la fiabilité des données recueillies sur des variables définies dans un référentiel différent. Ces caractères posent probablement moins de problèmes de mesure dans les pays développés.

Examinons à présent l'effet des variables de réforme à la lumière des résultats résumés dans le Tableau 4. Concernant le marché de la téléphonie mobile, les résultats montrent que l'accroissement de la concurrence entraine une augmentation du nombre d’abonnés et une baisse du tarif. De plus, ces relations sont robustes au vu des résultats des tests de Granger-causalité (voir en annexe, le Tableau III de la section C). La concurrence a donc bien produit les effets attendus en termes d'efficience allocative. Il convient toutefois de modérer quelque peu ce résultat et tenir compte du faible degré de satisfaction de la demande qui caractérisait vraisemblablement la plupart des pays en voie de développement, notamment au début de la période couverte par l’étude.

Des relations sont également mises en évidence entre le degré de privatisation de l’opérateur historique et la performance du segment de la téléphonie mobile, à savoir, une augmentation du nombre d’abonnés et du tarif. Surprenant, cet accroissement simultané de l'output et du prix consécutif à la privatisation de l’opérateur historique est un résultat mis en évidence dans la littérature (Gasmi et Recuero Virto, 2007 et 2009, Ross, 2009). Il reflète essentiellement la prévalence dans les pays en voie de développement d'une large demande non satisfaite (unmet demand).

Concernant le marché du fixe, les résultats montrent qu'une plus forte intensité concurrentielle se traduit par une baisse du tarif, ce qui conforte l'existence d'un effet 
bénéfique des variables de réforme sur la performance économique du secteur étudiée ${ }^{30}$. Les autres variables de contrôle (variables démographiques) ne produisent pas d'effets significatifs sur les indicateurs de performance et nous constatons aussi que les effets liés aux indicatrices du temps sont nettement moins présents que dans le cas du Panel 1.

D’une manière générale, les résultats mis en évidence dans ce groupe de pays semblent plus consistants que ceux obtenus dans celui des pays à revenus intermédiaires. En effet, les résultats du panel 2 font mieux ressortir le lien entre la qualité des institutions et la performance économique du secteur des télécommunications. Un effet plus significatif des variables de réforme sur la performance économique du secteur ressort également des résultats concernant ce groupe de pays. La quasi-absence de significativité des indicatrices du temps montre que la performance économique du secteur d'intérêt n'a pas tant été affectée par des événements spécifiques conjoncturels que par des facteurs plus structurels.

Tableau 4

Impact de la qualité institutionnelle sur la performance sectorielle (Panel 2 : pays à faibles revenus) $^{31}$

\begin{tabular}{|c|c|c|c|c|c|c|c|c|c|c|c|}
\hline \multicolumn{12}{|c|}{ Variables dépendantes (Indicateurs de performance) } \\
\hline \multicolumn{2}{|l|}{ Variables indépendantes } & \multicolumn{2}{|c|}{ tele-fix } & \multicolumn{2}{|c|}{ mobil } & \multicolumn{2}{|c|}{ l-emp } & \multicolumn{2}{|c|}{-fix } & \multicolumn{2}{|c|}{$p-m b l$} \\
\hline \multicolumn{2}{|l|}{ Environnement institutionnel } & NS & & + & & + & & + & & \multicolumn{2}{|l|}{-} \\
\hline \multicolumn{2}{|c|}{ Equilibre des pouvoirs } & NS & & - & & NS & & NS & & \multicolumn{2}{|l|}{ NS } \\
\hline \multicolumn{2}{|c|}{ privat } & NS & & + & & NS & & NS & & \multicolumn{2}{|l|}{+} \\
\hline \multicolumn{2}{|c|}{ concur-fix } & NS & & NS & & NS & & - & & \multicolumn{2}{|l|}{ NS } \\
\hline \multicolumn{2}{|c|}{ concur-mobil } & + & & + & & NS & & NS & & \multicolumn{2}{|l|}{-} \\
\hline \multicolumn{2}{|c|}{ sep-regul } & NS & & NS & & NS & & NS & & \multicolumn{2}{|l|}{ NS } \\
\hline \multicolumn{2}{|c|}{ dens-pop } & NS & & NS & & NS & & NS & & \multicolumn{2}{|l|}{ NS } \\
\hline \multicolumn{2}{|c|}{ pop-rural } & NS & & NS & & NS & & NS & & \multicolumn{2}{|l|}{ NS } \\
\hline \multicolumn{2}{|c|}{ Réformes endogènes } & Oui & & Oui & & Non & & Oui & & \multicolumn{2}{|l|}{ Oui } \\
\hline \multicolumn{12}{|c|}{ Indicatrices du temps } \\
\hline $\begin{array}{c}\text { Variables } \\
\text { dépendantes }\end{array}$ & 89 & 90 & 91 & 92 & 93 & 94 & 95 & 96 & 97 & 98 & 99 \\
\hline tele-fix & + & NS & NS & NS & NS & NS & NS & NS & NS & NS & NS \\
\hline$p$-fix & NS & NS & NS & NS & NS & - & NS & NS & NS & NS & NS \\
\hline \multicolumn{12}{|c|}{$\begin{array}{l}\text { NS : Non significatif au seuil de } 5 \% . \\
+: \text { : Effet positif et significatif au seuil de } 5 \% \text {. } \\
\text { - : Effet négatif et significatif au seuil de } 5 \% \text {. }\end{array}$} \\
\hline \multicolumn{12}{|c|}{$\begin{array}{l}{ }^{30} \text { Concernant l'impact des réformes, il est intéressant de souligner qu'alors que Gasmi et Recuero Virto (2007 } \\
\text { et 2009) et Ross (1999) mettent en évidence une relation empirique positive entre la privatisation et la } \\
\text { télédensité dans des bases de données agrégées sur les pays en voie de développement, dans le présent article } \\
\text { cette relation n'est significative que dans les données relatives aux pays du Panel 1. Ce résultat justifie a } \\
\text { posteriori la décomposition de notre base de données en deux panels. } \\
{ }^{31} \text { Le Tableau IV-b de l'Annexe (Section D) synthétise les résultats des régressions pour ce groupe de pays. }\end{array}$} \\
\hline
\end{tabular}


Il convient enfin de souligner le caractère endogène des variables de réforme qui ressort dans les résultats des tableaux 3 et 4 . L'interprétation que l'on peut en donner est que la privatisation et la libéralisation du secteur des télécommunications dans les deux groupes de pays n’apparaissent pas comme étant des mesures de politique économique "imposées" par des facteurs externes. Les réformes entreprises dans ces pays semblent plutôt être induites par des facteurs internes non observés (effets spécifiques individuels) ou par l'existence de pré requis (en termes de performance) à l’octroi de licences pour l'entrée de nouveaux opérateurs sur le marché.

\section{Conclusion}

Au vu des nombreuses études récentes mettant en évidence le rôle crucial que jouent les institutions dans le développement économique, dans cet article nous nous sommes interrogés sur la robustesse de cette relation aux différences de revenus qui existent entre les pays en voie de développement. Plus précisément, en décomposant une base de données sur ces pays en deux groupes selon le PIB par tête, nous avons testé l'hypothèse d'existence d'un seuil de revenus au-dessous duquel l'impact économique d’un effort d'amélioration de la qualité des institutions serait négligeable. La validation d’une telle hypothèse suggérerait la nécessité de différencier les pays en voie de développement selon leur richesse lorsqu’il s'agit d'évaluer les effets qu'on peut anticiper des politiques d'aide au renforcement des institutions. Cette hypothèse a été explorée pour le cas des télécommunications à l'aide d'une série de régressions de variables de performance économique de ce secteur sur des variables de qualité des institutions, en tenant compte de l'effet de variables traduisant les réformes mises en œuvre dans le secteur. L’étude a été menée à l'aide d’un modèle dynamique à effets fixes individuels spécifié suivant la méthode d'analyse des séries temporelles en coupes transversales.

L’analyse empirique n'a pas validé l'hypothèse d'existence d’un seuil rédhibitoire de pauvreté qui "stériliserait" des politiques visant le renforcement des institutions dans les pays les moins avancés. Bien au contraire, les résultats des régressions ont montré que, pour le cas des télécommunications, le lien entre la qualité des institutions et la performance économique du secteur est nettement plus significatif dans le groupe des pays à plus faibles revenus. Les régressions ont été effectuées en tenant compte d'un certain nombre de réformes qu’a connu le secteur, notamment la libéralisation des marchés et la privatisation de l'opérateur historique. Les résultats ont montré que ces variables sont également 
pertinentes pour expliquer les performances économiques du secteur dans les deux groupes de pays.

Un premier enseignement que l'on peut tirer de cette étude est que les institutions constituent bien un facteur clé du développement économique, particulièrement dans les pays les moins avancés. Nos résultats corroborent donc ceux des études antérieures qui établissent une relation étroite entre la qualité des institutions et le développement économique. Notre étude contribue ainsi à montrer la robustesse de cette relation au niveau de développement des pays.

Un autre enseignement que l'on peut tirer du résultat que le sous échantillons des pays à plus faibles revenus met davantage en évidence l’impact de la qualité des institutions sur la performance économique sectorielle est que le PIB par tête peut constituer un critère de différenciation des mesures de politique économique acceptable. Ce résultat plaide en faveur d’une réallocation des ressources consacrées à l'aide au développement au profit des mesures de renforcement des institutions dans les pays à faible PIB par tête.

Au-delà des résultats économétriques, l'étude a permis de prendre conscience de la difficulté de définir des variables capables de saisir de manière satisfaisante des caractères tels que la qualité des institutions. En effet, les résultats trouvés n’ont pas toujours été faciles à interpréter, certains d'entre eux étant contre-intuitifs parfois même contradictoires. Ainsi en est-il de la variable Equilibre des pouvoirs qui est censée refléter la qualité du processus politique. Le simple constat de l'existence d'institutions censées représenter les interactions entre les trois pouvoirs ne suffit en effet pas à apprécier la qualité du processus politique car il ne saisit pas le hiatus entre "cadre institutionnel formel" et "pratiques institutionnelles effectives", phénomène courant dans les pays en voie de développement.

Cette étude suggère plusieurs pistes de recherche future. Une première consisterait à élargir la définition des institutions utilisée dans les études économétriques menées à ce jour pour inclure les normes informelles les plus répandues dans le monde en voie de développement. Il nous semble également important de revenir sur les méthodes de collecte des données auprès des différents pays en s'efforçant d'observer des faits plutôt que de constater l'existence d'institutions censées produire ces faits. Par exemple, la compréhension et la prise en compte des distorsions pouvant exister entre institutions formelles et pratiques institutionnelles effectives permettrait de mieux apprécier la qualité du processus politique. Enfin, pour améliorer notre compréhension du rôle des institutions dans le développement économique des secteurs d'infrastructure, il serait intéressant d'explorer l'impact de leur qualité sur des aspects directement observables liés aux décisions des agents clés au 
développement de ces secteurs, notamment, les régulateurs, les dirigeants des opérateurs et les ministères de tutelle. 


\section{A. Sources des données statistiques}

Variables

Sources

\section{Performance du secteur}

\section{des télécommunications}

-Télédensité du réseau fixe

-Nombre d'abonnés au cellulaire

-Nombre de lignes fixes/employé

-Tarif du fixe

- ITU ; World Telecommunications Regulatory database

-Tarif du cellulaire

\section{Qualité de l'environnement} institutionnel

(Indice calculé par sommation des valeurs des 5 variables ci-dessous)

-Degré de corruption

-Qualité de l'appareil administratif -Force du système judiciaire et degré d'observance des lois

-Risque d'expropriation

-Risque de change
- Données produites par Steve Knack et Philip Keefer pour le centre IRIS de l'Université de Maryland (1982-1997)

- International Country Risk Guide (ICRG) risk ratings (1997-1999)

- IRIS dataset by Steve Knack and Philip Keefer for the IRIS Center at the University of Maryland (1982-1997)

- Exchange Rate Stability, International Country Risk Guide (ICRG) risk ratings (1985-1999)

\section{Qualité du processus politique}

-Equilibre des pouvoirs

\section{Réformes}

-Degré d’ouverture du capital de l’opérateur historique

-Libéralisation du fixe

-Libéralisation du mobile

-Séparation du régulateur
- DPI 2000 Database of Political Institutions 19752000, Philip Keefer (Development Research Group), The World Bank (2002)
- Ros (1999, 2003), Bortolotti et al. (2001), McNary (2001), Li and Xu (2004), Fink et al. (2002), Clark et al. (2004)

- ITU World Telecoms Regulatory database

- Sites web : opérateurs et autorités de régulation

- Private Partcipation in Infrastructure (PPI), Project World Bank database

- IPANeT Privatization Transactions database-WB 
- Ros (1999, 2003), Bortolotti et al. (2001), McNary (2001), Li and Xu (2004), Fink et al. (2002), Clark et al. (2004)

- ITU World Telecoms Regulatory database

- Sites web : opérateurs et autorités de régulation

- http://www.gsmworld.com

- Bortolotti et al. (2001), Fink et al. (2002),

- Gutierrez (2003), Ros (2003)

- ITU World Telecommunications Regulatory database

- Clark et al. (2004)

\section{Démographie}

-Densité de la population globale

-Poids de la population rurale

- Worldbank.org ; datastatistics

- Population and Demographic Indicators

- World development indicators

\section{B. Définition des variables du modèle}

\section{B.1. Variables dépendantes}

- tele-fix (télédensité du fixe) : nombre de lignes téléphoniques pour 100 habitants.

- mobil : nombre d’utilisateurs de téléphones portables, abonnés à un service de téléphonie mobile.

- l-emp : nombre de lignes principales divisé par le nombre d'employés dans le secteur de téléphonie fixe.

- p-fix (Tarif de la téléphonie fixe) : charge mensuelle d'accès au réseau filaire (hors charges d'utilisation du terminal), exprimée en dollars US de 2000.

- p-mobil (Tarif de la téléphonie mobile) : prix payé pour un appel de trois minutes durant les heures de pointe à partir d'un téléphone portable en utilisant une carte prépayée (en dollars US de 2000). 


\section{B.2. Variables indépendantes}

\section{B.2.1. Indicateurs institutionnels}

- corrup (Indice de corruption) : caractère mesuré sur une échelle discrète de 0 à 10 . Des valeurs plus élevées indiquent un plus faible degré de corruption du système politique.

- adm (Qualité de l'appareil administratif de l'Etat) : caractère mesuré sur une échelle de 0 à 10. Des scores élevés sont attribués aux pays où le système administratif possède la capacité de gouverner sans recourir à des changements drastiques dans les politiques en vigueur.

- loi (Loi et ordre public) : caractère mesuré sur une échelle de 0 à 10 , pour évaluer la force et l'impartialité du système judiciaire en place ainsi que le degré d’observance de la loi par les citoyens. Des scores plus élevés indiquent une meilleure qualité du système judiciaire en place.

- exprop (Risque d'expropriation des investisseurs privés, par dépossession ou nationalisation) : caractère mesuré sur une échelle de 0 à 10 , les valeurs plus élevées reflétant un risque d'expropriation plus faible.

- change (Risque de change) : caractère mesuré sur une échelle de 0 à 10 Des valeurs plus élevées reflètent un moindre risque de change.

- inst (Qualité de l’environnement institutionnel) : Indice établi par sommation verticale des cinq variables décrites ci-dessus, d'où une échelle de 0 à 50. Des valeurs supérieures indiquent une meilleure qualité institutionnelle.

\section{B.2.2. Qualité du processus politique}

- pouvoir (Equilibre des pouvoirs) : caractère mesuré sur une échelle de 0 à 18 . Des valeurs plus élevées indiquent un meilleur fonctionnement du processus politique d'ensemble.

\section{B.2.3. Variables de réforme}

- privat : pourcentage des actifs de l’Opérateur historique cédés aux investisseurs privés. 
- concur-fix (Concurrence sur le fixe) : indicatrice prenant la valeur 0 si la structure de marché au niveau local est de type monopolistique et la valeur 1 si ce segment met en jeux deux opérateurs ou plus.

- concur- mobil (Concurrence sur le cellulaire) : indicatrice prenant la valeur 0 si aucune licence n’a été délivrée, les valeurs 1 ou 2 si une ou deux licences ont été attribuées et la valeur 3 si trois licences ou plus ont été délivrées.

- sep-regul : indicatrice prenant la valeur 1 s’il y a séparation de l'instance de régulation sectorielle et 0 si non.

\section{B.2.4. Variables démographiques}

- dens-pop (densité de la population) : Nombre d’habitants au Km².

- pop-rural (Importance de la population rurale) : proportion des habitants résidant en zones rurales. 


\section{Tableaux statistiques des résultats (stationnarité et causalité)}

Tableau I

Tests de stationnarité des indicateurs de performance

(Panel 1 : pays à revenus intermédiaires)

\begin{tabular}{|c|c|c|c|c|}
\hline $\ln \left(t e l e-f i x_{\mathrm{it}}\right)$ & DIF-GMM & SYS-GMM & $\Delta \ln \left(\right.$ tele-fix $\left.x_{\mathrm{it}}\right)$ & DIF-GMM \\
\hline $\ln \left(\right.$ tele-fix $\left.x_{\mathrm{it}-1}\right)$ & $0.799^{\mathrm{s}}$ & $0.979^{\mathrm{s}}$ & $\Delta \ln \left(r-l p_{\mathrm{it}-1}\right)$ & $0.474^{\mathrm{s}}$ \\
\hline Trend & $0.017^{\mathrm{s}}$ & $0.005^{\mathrm{s}}$ & Trend & 0.001 \\
\hline$m_{1}$ & -1.85 & $-2.12^{\mathrm{s}}$ & $m_{1}$ & $-2.82^{s}$ \\
\hline$m_{2}$ & 0.37 & 0.24 & $m_{2}$ & 1.53 \\
\hline$J$-stat & 14.44 & 12.96 & $J$-stat & 13.57 \\
\hline$L$ & 2 & 2 & $L$ & 2 \\
\hline Nombre Obs. & 208 & 224 & Nombre Obs. & 208 \\
\hline $\ln \left(\right.$ mobil $\left._{\mathrm{it}}\right)$ & DIF-GMM & SYS-GMM & $\Delta \ln \left(m_{o b i l}\right)$ & DIF-GMM \\
\hline $\ln \left(\right.$ mobil $\left._{\mathrm{it}-1}\right)$ & $1.038^{\mathrm{s}}$ & $1.043^{\mathrm{s}}$ & $\Delta \ln \left(\right.$ mobil $\left._{\mathrm{it}-1}\right)$ & 0.201 \\
\hline Trend & $0.029^{s}$ & $0.038^{\mathrm{s}}$ & Trend & $0.032^{\mathrm{s}}$ \\
\hline$m_{1}$ & -1.63 & -1.60 & $m_{1}$ & $-2.52^{\mathrm{s}}$ \\
\hline$m_{2}$ & -0.18 & -0.10 & $m_{2}$ & 0.78 \\
\hline$J$-stat & 14.35 & 9.96 & $J$-stat & 15.53 \\
\hline$L$ & 2 & 2 & $L$ & 2 \\
\hline \multirow[t]{17}{*}{ Nombre Obs. } & 200 & 217 & Nombre Obs. & 184 \\
\hline & $\ln \left(p-m o b i l_{\text {it }}\right)$ & DIF-GMM & SYS-GMM & \\
\hline & $\ln \left(p-m o b i l_{\mathrm{it}-1}\right)$ & $0.445^{\mathrm{s}}$ & $0.941^{\mathrm{s}}$ & \\
\hline & Trend & $-0.011^{\mathrm{s}}$ & -0.001 & \\
\hline & $m_{1}$ & -1.45 & -1.75 & \\
\hline & $m_{2}$ & -0.63 & -0.72 & \\
\hline & $J$-stat & 8.87 & 12.10 & \\
\hline & $L$ & 2 & 2 & \\
\hline & Nombre Obs. & 116 & 142 & \\
\hline & $\ln \left(p-f i x_{\mathrm{it}}\right)$ & DIF-GMM & SYS-GMM & \\
\hline & $\ln \left(p-f i x_{\mathrm{it}-1}\right)$ & $0.694^{\mathrm{s}}$ & $0.818^{\mathrm{s}}$ & \\
\hline & Trend & 0.008 & 0.006 & \\
\hline & $m_{1}$ & -1.79 & $-2.01^{\mathrm{s}}$ & \\
\hline & $m_{2}$ & 0.20 & 0.22 & \\
\hline & $J$-stat & 11.71 & 11.81 & \\
\hline & $L$ & 2 & 2.00 & \\
\hline & Nombre Obs. & 97 & 112 & \\
\hline $\ln \left(l-e m p_{\mathrm{it}}\right)$ & DIF-GMM & SYS-GMM & $\Delta \ln \left(\right.$ l-emp $\left.p_{i t}\right)$ & DIF-GMM \\
\hline $\ln \left(l-e m p_{i t-1}\right)$ & $1.017^{\mathrm{s}}$ & $1.015^{\mathrm{s}}$ & $\Delta \ln \left(\right.$ l-emp $\left.{ }_{\mathrm{it}-1}\right)$ & 0.131 \\
\hline Trend & --- & --- & Trend & --- \\
\hline$m_{1}$ & $-2.79^{s}$ & $-2.78^{s}$ & $M_{1}$ & $-3.22^{s}$ \\
\hline$m_{2}$ & -0.24 & -0.25 & $M_{2}$ & -0.07 \\
\hline$J$-stat & 15.98 & 15.98 & $J$-stat & 13.83 \\
\hline$L$ & 2 & 2 & $L$ & 2 \\
\hline Nombre Obs. & 198 & 216 & Nombre Obs. & 181 \\
\hline
\end{tabular}

S : Significatif au seuil de $5 \%$. 
Tableau II

Tests de stationnarité des indicateurs de performance

(Panel 2 : pays à faibles revenus)

\begin{tabular}{|c|c|c|c|c|}
\hline $\ln \left(\right.$ tele-fix $\left.x_{i t}\right)$ & DIF-GMM & SYS-GMM & $\Delta \ln \left(\right.$ tele-fix $\left.x_{i t}\right)$ & DIF-GMM \\
\hline $\ln \left(\right.$ tele-fix $\left.x_{\mathrm{it}-1}\right)$ & $0.927^{\mathrm{s}}$ & $1.041^{\mathrm{s}}$ & $\begin{array}{l}\Delta \ln (\text { tele-fix } \\
\left.{ }_{1}\right)\end{array}$ & $0.632^{\mathrm{s}}$ \\
\hline Trend & $0.009^{\mathrm{s}}$ & $0.003^{\mathrm{s}}$ & Trend & 0.002 \\
\hline$m_{1}$ & -1.59 & -1.63 & $m_{1}$ & $-2.21^{\mathrm{s}}$ \\
\hline$m_{2}$ & 1.12 & 1.07 & $m_{2}$ & 1.62 \\
\hline$J$-stat & 12.88 & 13.29 & $J$-stat & 11.54 \\
\hline$L$ & 2 & 2 & $L$ & 2 \\
\hline Nombre Obs. & 208 & 224 & Nombre Obs. & 208 \\
\hline $\ln \left(m_{0 b i l}\right)$ & DIF-GMM & SYS-GMM & $\Delta \ln \left(m_{0 b i l}\right)$ & DIF-GMM \\
\hline $\ln \left(\right.$ mobil $\left._{i t-1}\right)$ & $1.004^{\mathrm{s}}$ & $1.075^{\mathrm{s}}$ & $\left.\Delta \ln \left(m^{2} b_{i t}\right)_{i t-1}\right)$ & $0.690^{\mathrm{s}}$ \\
\hline Trend & $0.028^{\mathrm{s}}$ & $0.026^{\mathrm{s}}$ & Trend & $0.018^{\mathrm{s}}$ \\
\hline$m_{1}$ & 0.55 & 0.31 & $m_{1}$ & -1.85 \\
\hline$m_{2}$ & -0.07 & -0.41 & $m_{2}$ & -0.68 \\
\hline$J$-stat & 14.26 & 14.88 & $J$-stat & 14.83 \\
\hline$L$ & 2 & 2 & $L$ & 2 \\
\hline \multirow[t]{17}{*}{ Nombre Obs. } & 208 & 224 & Nombre Obs. & 208 \\
\hline & $\ln \left(p-\right.$ mobil $\left._{i t}\right)$ & DIF-GMM & SYS-GMM & \\
\hline & $\ln \left(p-\right.$ mobil $\left._{\mathrm{it}-1}\right)$ & 0.377 & $0.939^{\mathrm{s}}$ & \\
\hline & Trend & $-0.043^{s}$ & -0.05 & \\
\hline & $m_{1}$ & -0.64 & -1.37 & \\
\hline & $m_{2}$ & 1.49 & -0.07 & \\
\hline & $J$-stat & 7.55 & 2.01 & \\
\hline & $L$ & 2 & 2 & \\
\hline & Nombre Obs. & 115 & 138 & \\
\hline & $\ln \left(p-f i x_{i t}\right)$ & DIF-GMM & SYS-GMM & \\
\hline & $\ln \left(p-f i x_{i t-1}\right)$ & $0.544^{\mathrm{s}}$ & $0.865^{\mathrm{s}}$ & \\
\hline & Trend & $-0.23^{\mathrm{s}}$ & -0.07 & \\
\hline & $m_{1}$ & $-2.02^{\mathrm{s}}$ & -1.84 & \\
\hline & $m_{2}$ & 0.17 & 0.19 & \\
\hline & $J$-stat & 12.58 & 14.52 & \\
\hline & $L$ & 2 & 2 & \\
\hline & Nombre Obs. & 116 & 134 & \\
\hline $\ln \left(l-e m p_{\mathrm{it}}\right)$ & DIF-GMM & SYS-GMM & $\Delta \ln \left(l-e m p_{i t}\right)$ & DIF-GMM \\
\hline $\ln \left(\right.$ l-emp $\left.p_{\mathrm{it}-1}\right)$ & $0.782^{\mathrm{s}}$ & $0.984^{\mathrm{s}}$ & $\Delta \ln \left(\right.$ l-emp $\left.{ }_{\mathrm{it}-1}\right)$ & 0.008 \\
\hline Trend & $0.023^{\mathrm{s}}$ & $0.08^{\mathrm{s}}$ & Trend & 0.004 \\
\hline$m_{1}$ & $-2.26^{\mathrm{s}}$ & $-2.10^{\mathrm{s}}$ & $m_{1}$ & $-2.73^{\mathrm{s}}$ \\
\hline$m_{2}$ & -1.48 & -1.45 & $m_{2}$ & -0.99 \\
\hline$J$-stat & 12.96 & 10.93 & $J$-stat & 13.03 \\
\hline$L$ & 2 & 2 & $L$ & 2 \\
\hline Nombre Obs. & 199 & 216 & Nombre Obs. & 199 \\
\hline
\end{tabular}

S : Significatif au seuil de $5 \%$. 
Tableau III

Tests de causalité

(Panel 2 : pays à faibles revenus) ${ }^{32}$

\begin{tabular}{|c|c|c|c|c|}
\hline Variables indépendantes & $\underset{\left(\mathrm{y}_{\mathrm{it}}\right)}{\Delta \ln m o b i l_{i t}}$ & $\begin{array}{c}\text { Variables } \\
\text { indépendantes }\end{array}$ & $\begin{array}{c}\Delta \ln \text { mobil }_{i t} \\
\left(\mathrm{y}_{\mathrm{it}}\right)\end{array}$ & $\begin{array}{c}\Delta \ln p-\text { mobil }_{i t} \\
\left(\mathrm{y}_{\mathrm{it}}\right)\end{array}$ \\
\hline$y_{i t-1}$ & $.3992^{\mathrm{s}}$ & $y_{i t-1}$ & $.5745^{\mathrm{s}}$ & .0988 \\
\hline$y_{i t-2}$ & -.0747 & $y_{i t-2}$ & -.1336 & \\
\hline$y_{i t-3}$ & .1621 & inst $_{i t-1}$ & $.0089^{\mathrm{s}}$ & $-.0328^{\mathrm{s}}$ \\
\hline pouvoir $_{\text {it-1 }}$ & .0006 & inst $_{i t-2}$ & $-.0082^{\mathrm{s}}$ & \\
\hline pouvoir $_{i t-2}$ & -.0063 & pouvoir & -.0012 & -.0104 \\
\hline pouvoir $_{i t-3}$ & $-.0292^{\mathrm{s}}$ & concur-fix ${ }_{i t}$ & .0259 & $.6537^{\mathrm{s}}$ \\
\hline corrup $_{\text {it }}$ & .0042 & concur-mobil ${ }_{i t}$ & $.1009^{\mathrm{s}}$ & $-.1522^{\mathrm{s}}$ \\
\hline loi & $.0118^{\mathrm{s}}$ & sep-regul & .0569 & $-.1083^{s}$ \\
\hline Adm & .0559 & privat & .1546 & $-.2999^{\mathrm{s}}$ \\
\hline exprop & $-.0223^{s}$ & & & \\
\hline concur-fix $x_{i t}$ & .0135 & & & \\
\hline concur-mobil $_{i t}$ & $.1455^{\mathrm{s}}$ & & & \\
\hline privat & .1445 & & & \\
\hline change & $.0171^{\mathrm{s}}$ & & & \\
\hline sep-regul & -.0286 & & & \\
\hline$m_{1}$ & -2.12 & $m_{1}$ & -2.69 & 0.38 \\
\hline$m_{2}$ & -1.05 & $m_{2}$ & 0.35 & 0.99 \\
\hline$J$-stat & 0.00 & $J$-stat & 4.13 & 4.20 \\
\hline$L$ & 2 & $L$ & 2 & 2 \\
\hline Nombre d'Obs. & 152 & Nombre d'Obs. & 168 & 115 \\
\hline$m^{\mathrm{a}}$ & 3 & $m$ & 2 & 1 \\
\hline$m$-test ${ }^{\mathrm{b}}$ & $13.22^{\mathrm{s}}$ & $m$-test & $5.09^{\mathrm{s}}$ & $33.10^{\mathrm{s}}$ \\
\hline Causalité $^{\mathrm{c}}$ & $17.11^{\mathrm{s}}$ & Causalité & $5.49^{\mathrm{s}}$ & $16.22^{\mathrm{s}}$ \\
\hline
\end{tabular}

a : $m$ désigne la longueur de décalage maximale dans l'équation (3), utilisée pour les tests de causalité au sens de Granger.

b : Statistique de Wald pour tester la significativité des coefficients de l'équation (3)..

c : Statisiique de Walgd..

S : Siginficatif au seuil de $5 \%$.

${ }^{32}$ Pour le Panel 1, nous n'avons pas obtenu de résultats significatifs pour la causalité au sens de Granger. Concernant le Panel 2, seules les variables pour lesquelles des liens de causalité au sens de Granger ont été trouvés apparaissent dans le Tableau III. 


\section{Tableaux statistiques des résultats (régressions)}

Tableau IV-a

Paramètres estimés / Modèle DIF-GMM

(Panel 1 : pays à revenus intermédiaires)

\begin{tabular}{|c|c|c|c|c|c|c|}
\hline \multirow{2}{*}{\multicolumn{2}{|c|}{ Variables indépendantes }} & \multicolumn{5}{|c|}{ Variables dépendantes $\left(y_{i t}\right)$} \\
\hline & & tele-fix $x_{i t}$ & mobil $_{i t}$ & l-emp $p_{i t}$ & $p-f_{i x}$ & $p$-mobil ${ }_{i t}$ \\
\hline \multicolumn{2}{|r|}{$y_{i t-1}$} & .1529 & $.9560^{\mathrm{s}}$ & -.0300 & $.6020^{\mathrm{s}}$ & $.5279^{\mathrm{s}}$ \\
\hline \multicolumn{2}{|r|}{ inst $_{i t}$} & -.0014 & .0005 & -.0023 & $-.0346^{s}$ & .0093 \\
\hline \multicolumn{2}{|r|}{ pouvoir $_{i t}$} & .0071 & -.0214 & -.0032 & -.0099 & .0113 \\
\hline \multicolumn{2}{|r|}{ concur-fix $x_{i t}$} & $-.0635^{s}$ & -.0486 & $-.1539^{s}$ & .0447 & -.0121 \\
\hline \multicolumn{7}{|c|}{ concur- } \\
\hline \multicolumn{2}{|r|}{ mobil $_{i t}$} & .0065 & $.0975^{\circ}$ & -.0145 & .0018 & .0655 \\
\hline \multicolumn{2}{|r|}{ sep-regul $_{i t}$} & .0013 & -.0505 & -.0014 & -.1110 & .1084 \\
\hline & & $.0902^{\mathrm{s}}$ & $.2751^{\mathrm{s}}$ & $.2599^{\mathrm{s}}$ & -.0369 & -.2401 \\
\hline \multicolumn{2}{|r|}{ dens-pop $_{i t}$} & .0005 & .0108 & $.0075^{\mathrm{s}}$ & $.0248^{\mathrm{s}}$ & -.0098 \\
\hline \multicolumn{2}{|r|}{ pop-rural $_{\text {it }}$} & $-.0107^{\mathrm{s}}$ & .0175 & $-.0163^{s}$ & $.1052^{\mathrm{s}}$ & .0178 \\
\hline \multirow{12}{*}{$\begin{array}{l}\text { Indicatrices } \\
\text { du temps }\end{array}$} & 1988 & -.0023 & $.1428^{\mathrm{s}}$ & -- & -- & -- \\
\hline & 1989 & -.0240 & $.0647^{\mathrm{s}}$ & -- & -- & -- \\
\hline & 1990 & -.0119 & $.0925^{\mathrm{s}}$ & -- & -- & -- \\
\hline & 1991 & $-.0299^{\mathrm{s}}$ & .0663 & -- & -- & -- \\
\hline & 1992 & -.0101 & $.0934^{\mathrm{s}}$ & -.0076 & $.4438^{\mathrm{s}}$ & -- \\
\hline & 1993 & -.0306 & $.1092^{\mathrm{s}}$ & -.0277 & $.3476^{\mathrm{s}}$ & -- \\
\hline & 1994 & -.0172 & .1265 & .0116 & $.4771^{\mathrm{s}}$ & -- \\
\hline & 1995 & $-.0502^{\mathrm{s}}$ & $.1640^{\mathrm{s}}$ & -.0002 & $.5653^{\mathrm{s}}$ & -- \\
\hline & 1996 & -.0315 & .1514 & .0036 & $.6422^{\mathrm{s}}$ & -- \\
\hline & 1997 & -.0293 & .1912 & -.0499 & $.7272^{\mathrm{s}}$ & -- \\
\hline & 1998 & $-.0712^{\mathrm{s}}$ & .2302 & -.1339 & $.6302^{\mathrm{s}}$ & -- \\
\hline & 1999 & $-.0679^{\mathrm{s}}$ & $.2658^{\mathrm{s}}$ & -.1178 & $.6542^{\mathrm{s}}$ & -- \\
\hline \multicolumn{2}{|l|}{$m_{1}$} & $-2.87^{\mathrm{s}}$ & $-2.85^{\mathrm{s}}$ & $-2.95^{\mathrm{s}}$ & -1.93 & $-2.13^{s}$ \\
\hline \multicolumn{2}{|l|}{$m_{2}$} & 0.38 & $2.21^{\mathrm{s}}$ & -0.99 & 0.13 & -0.29 \\
\hline \multicolumn{2}{|l|}{$J$-stat } & 0.77 & 0.05 & 0.00 & 0.00 & 8.45 \\
\hline \multicolumn{2}{|l|}{$L$} & 2 & 2 & 2 & 2 & 2 \\
\hline \multicolumn{2}{|c|}{ Nombre d'Obs. } & 185 & 177 & 174 & 90 & 111 \\
\hline \multicolumn{2}{|c|}{ Réformes endogènes } & Oui & Oui & Oui & Oui & Oui \\
\hline
\end{tabular}

$\mathrm{S}$ : Significatif au seuil de $5 \%$. 
Tableau IV-b

Paramètres estimés / Modèle DIF-GMM

(Panel 2 : pays à faibles revenus)

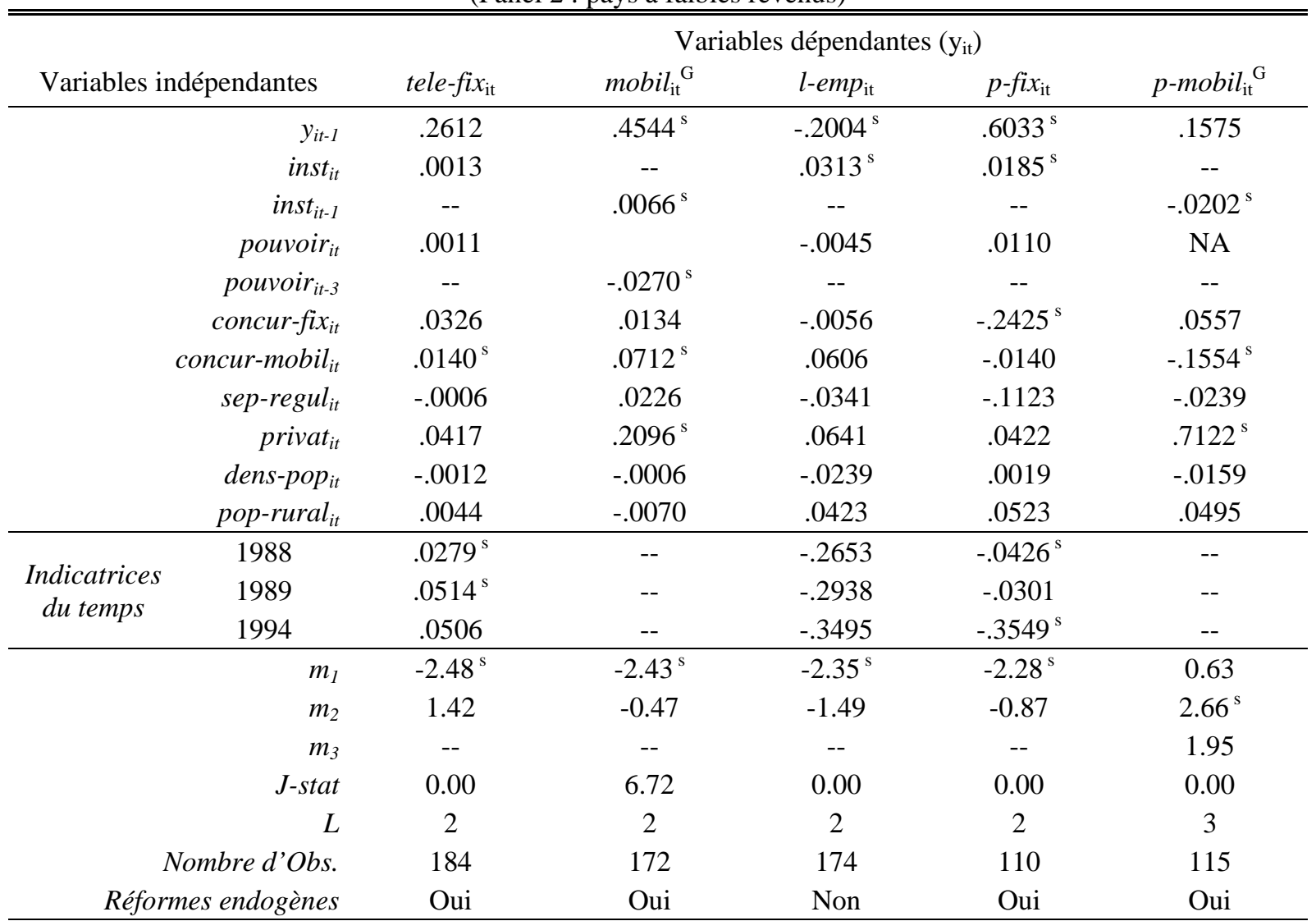

S : Significatif au seuil de $5 \%$.

$\mathrm{G}$ : Résultat trouvé avec test préalable de causalité au sens de Granger.

NA : Non applicable. 


\section{Références bibliographiques}

Alonso-Borrego, C. et M. Arellano, 1996, "Symmetrically normalized instrumental variable estimation using panel data", CEMFI Working Paper 9612.

Anderson, T. W. et C. Hsiao, 1981, "Estimation of dynamic model with error components", Journal of the American Statistical Association, 76: 598-606.

, 1982, "Formulation and estimation of dynamic model using panel data", Journal of econometrics, 18: 47-82.

Arellano, M. et S.R. Bond, 1991, "Some tests of specification for panel data: Monte Carlo evidence and an application to employment equations", Review of Economic Studies, 58: 277-297.

Blundell, R. et S.R. Bond, 1998, "Initial conditions and moment restrictions in dynamic panel data models", Journal of Econometrics, 87: 115-143.

Bortolotti, B., Fantini, M et S. Domenico, 2001. "Privatisation Around the World: New Evidence from Panel Data," CESifo Working Paper

Clark, G., Haggarty, L., Kaneshiro, R., Noll, G.R., Shirley, M., Wallsten, S., et L.C. Xu, 2004, "New tools for studying network industry reforms in developing countries: The telecommunications and electricity regulation database", AEI-Brookings Joint Center Related Publication 04-05

Cubbin, J. et J. Stern, 2005, "Regulatory effectiveness and the empirical impact of variations in regulatory governance: Electricity industry capacity and efficiency in developing countries", World Bank Policy Research Working Paper 3535.

Edison, H., 2003, "Qualité des institutions et résultats économiques - Un lien vraiment étroit?", Finances \& Développement, Juin : 35-37. 
Fink, C., Mattoo, A. et Rathindran, R., 2002, "An Assessment of Telecommunications Reform in Developing Countries", World Bank Policy Research Working Paper 2909.

Gasmi, F., Noumba, P., et L. Recuero Virto, 2006, "Political accountability and regulatory performance in infrastructure industries: An empirical analysis", World Bank Policy Research Paper 4101. A paraître dans The World Bank Economic Review.

Gasmi, F. et L. Recuero Virto, 2007, "The determinant and impact of telecommunications reforms in developing countries", Document de travail TSE, Université Toulouse 1 Capitole. A paraître dans Journal of Development Economics.

, 2009, "The impact of privatization of traditional fixed-line operators", Document de travail OCDE, Box 14, p. 108, African Economic Outlook, Part Two: "Innovation and ICT in Africa", OECD (2009).

Granger, C., 1969, "Investigating causal relations by econometric models and cross-spectral methods", Econometrica, 37 (3): 424-438.

Gutierrez, L. H., 2003, "Regulatory governance in the Latin American telecommunications sector," Utilities Policies, 11: 225-240

Holtz-Eakin, D., Newey, W. et H.S. Rosen, 1988, "Estimating vector auto regressions with panel data", Econometrica, 56: 1371-1395.

Levy, B. et P. Spiller, 1994, "The institutional foundations of regulatory commitment : a comparative analysis of telecommunications regulation", The Journal of Law, Economics, and Organization; 10: 201-246

Li, W. et C. L. Xu, , 2004, "The impact of privatization and competition in the telecommunications sector around the world", The Journal of Law and Economics, 47: 395-430

McNary, R., 2001, "The network penetration effects of telecommunications privatization and competition", Mimeo, Stanford University 
North, D. C., 1991, "Institutions", Journal of Economic Perspectives, 5: 97-112.

Roodman, D., 2005, "xtabond2: Stata Module to Extend xtabond Dynamic Panel Data Estimator", Document du Center for Global Development, Washington, DC.

Ros, A.J., 1999, "Does ownership and competition matter? The effects of telecommunications reform on network expansion and efficiency", Journal of Regulatory Economics, 15: 62-92

Ros, A.J., 2003, "The impact of the regulatory process and price cap regulation in Latin American telecommunications markets," Review of Network Economics, 2(3): 270-286

Sevestre, P, 2002, Econométrie des données de panel, Dunod, pp. 109-152.

et A. Trognon, 1996, Dynamic linear models in the econometrics of panel data, ch. 7, Kluwer Academic Publishers

Spiller, P.T., Stein, E. et M. Tommasi, 2003, "Political institutions, policymaking processes and policy outcomes. An inter temporal transactions framework", Design Paper DP1 for the project Political institutions, policymaking processes and policy outcomes.

Spiller, P.T. et M. Tommasi, 2003, "The institutions of regulation: An application to public utilities", Handbook of Telecommunications Economics, Vol. 2.

2007, The institutional foundations of public policy in Argentina - A transactions cost approach, Cambridge University Press.

Spiller, P.T. et S. Liao, 2008, "Buy, lobby or sue: Interest groups' participation in policy making - A selective survey", dans New Institutional Economics-A guide book, Cambridge University Press par E. Brousseau et J-M Glachant. 